\title{
DIVERSIDAD Y PATRONES DE DISTRIBUCIÓN DE LAS MARIPOSAS DIURNAS (LEPIDOPTERA: PAPILIONOIDEA Y HESPERIOIDEA) EN UN TRANSECTO ALTITUDINAL DEL PARQUE NACIONAL SIERRA MARTÍN GARCÍA, REPÚBLICA DOMINICANA
}

\author{
${ }^{1}$ Katihusca Rodríguez, ${ }^{1}$ Francisco Paz y ${ }^{2}$ Ruth H. Bastardo
}

${ }^{1}$ Museo Nacional de Historia Natural "Prof. Eugenio de Jesús Marcano" (MNHNSD). Calle César Nicolás Penson, Plaza de la Cultura Juan Pablo Duarte, Santo Domingo, República Dominicana. k.rodriguez@mnhn.gov.do,f.paz@mnhn.gov.do

${ }^{2}$ Instituto de Investigaciones Botánicas y Zoológicas Prof. Rafael M. Moscoso, Universidad Autónoma de Santo Domingo, Santo Domingo, República Dominicana.r_bastardo@hotmail.com

\section{RESUMEN}

Se evaluaron la diversidad y los patrones de distribución de la fauna de lepidópteros diurnos (Papilionoidea y Hesperioidea) en relación con los tipos de vegetación en un transecto altitudinal: 3-1,343 metros sobre el nivel del mar (msnm) del Parque Nacional Sierra Martín García, República Dominicana, Isla La Hispaniola. Se establecieron seis zonas de estudio correspondientes a sendos tipos de vegetación en los que se realizaron recorridos de una hora, con un total de 24 recorridos. Se reconocieron 71 especies, incluyendo 22 nuevos registros para la localidad. Los mayores valores de diversidad correspondieron a los bosques mesófilos que están a mayor altitud, los bosques húmedo y nublado, con un índice de Shannon de 2.72 y 2.60 , respectivamente. La vegetación ribereña mostró el mayor valor de dominancia de acuerdo al índice de Simpson $(\lambda=0.41)$. El índice de similitud de Jaccard evidenció un patrón altitudinal en la distribución de las comunidades encontrándose mayor similitud entre la fauna de zonas bajas del monte espinoso subtropical y el bosque seco.

Palabras clave: riqueza, diversidad, distribución, Lepidoptera, Sierra Martín García, República Dominicana.

Title: Diversity and distribution patterns of butterflies (Lepidoptera: Papilionoidea y Hesperioidea) in an altitudinal transect in Sierra Martín García National Park, Dominican Republic.

\section{ABSTRACT}

The diversity and distribution patterns of diurnal Lepidoptera (Papilionoidea and Hesperioidea), in relation with vegetation types in an altitudinal transect of 3-1343 meters above sea level (m.a.s.l.) of Sierra Martín García National Park, Dominican Republic, Hispaniolan Island, were evaluated. Six study zones, corresponding to six types of vegetation were established in which one hour trails were performed, with a total of 24 trails. A total of 71 species were documented, including 22 new records for the locality. Rain forest and cloud forest showed the highest diversity values (2.72 and 2.60, respectively) for the Shannon Index. The highest values for Simpson index were observed in the riparian forest $(\lambda=0.41)$. The Jaccard similarity index showed an altitudinal pattern in the distribution of communities with more similarity between the fauna of lowland subtropical thorn scrub and dry forest.

Keywords: richness, diversity, distribution, Lepidoptera, Sierra Martín García, Dominican Republic. 


\section{INTRODUCCIÓN}

La fauna de lepidópteros diurnos (Papilionoidea y Hesperioidea) de la Isla Hispaniola es de las mejores estudiadas de las Antillas Mayores, con un vasto historial de exploración e investigación taxonómica (Schwartz, 1983, 1987,1989; Smith et al., 1994, Takizawa et al., 2003; Perez-Gelabert, 2008; Sourakov y Zakharov, 2011). Para la República Dominicana, porción oriental de la isla, entomólogos locales han realizado estudios con un enfoque ecológico y conservacionista donde se incluyen las mariposas diurnas por su importancia en la determinación del grado de perturbación de los ecosistemas (Abreu y Guerrero, 1997; Navarro, 2002; Bastardo, 2007), sentando las bases para la consideración de los lepidópteros como grupo taxonómico de importancia ecológica en el país.

Smith et al. (1994) estiman unas 350 especies de mariposas diurnas para las Antillas. De esta región, Cuba, la isla de mayor tamaño $\left(109,890 \mathrm{~km}^{2}\right)$, posee la mayor riqueza de especies, 195 (Núñez Águila y Barro Cañamero, 2012). Sin embargo, La Hispaniola, segunda en tamaño con $76,480 \mathrm{~km}^{2}$ de extensión territorial, tiene una riqueza muy similar a la de Cuba, con un registro de 190 especies (Pérez-Gelabert, 2008; Sourakov y Zakaharov, 2011). Esta riqueza se debe en gran parte a que La Hispaniola es una isla con una gran variedad de ambientes, incluyendo ecosistemas únicos en el Caribe, extensos bosques de pino (Pinus occidentalis Sw.) en la Cordillera Central, la mayor elevación de todo el Caribe $(3,175 \mathrm{msnm})$ y extensos bosques secos a baja elevación. Una diversidad similar se encuentra en vertebrados (Myers et al., 2000; Powell e Incháustegui, 2009; Perdomo et al., 2010).

Este trabajo compara los resultados de expediciones recientes a la Sierra Martín García con datos históricos (Schwartz, 1987) y analiza diversos aspectos de la diversidad y patrones de distribución de las mariposas diurnas en ese sistema montañoso.

\section{OBJETIVO}

- Evaluar la diversidad y distribución de la fauna de lepidópteros diurnos en un transecto altitudinal en el Parque Nacional Sierra Martín García.

\section{MATERIALES Y MÉTODOS}

Área de estudio. La Sierra Martín García (SMG), se encuentra en el suroeste de la República Dominicana. Es un macizo montañoso de topografía accidentada que se eleva frente a las costas de la Bahía de Neiba, entre las provincias de Azua y Barahona. Las altitudes varían entre el nivel del mar y los 1,343 msnm en Loma del Curro (De La Fuente, 1976; Troncoso, 1986). Esta variación altitudinal ha permitido el desarrollo de varios tipos de vegetación (García et al., 2007): en zonas áridas de menor elevación (3-600 msnm), el monte espinoso subtropical (MES) y el bosque seco (BS); bosques de transición de seco a húmedo en las zonas bajas y medias entre 600-800 msnm (BT); bosque húmedo $(\mathrm{BH})$ y bosque nublado $(\mathrm{BN})$ en la parte más alta de la sierra; la vegetación ribereña (VR) ocupa diferentes alturas dependiendo de la localidad en la que se encuentre.

Muestreo. Los muestreos se realizaron en dos áreas de la sierra, la primera comprendió desde la carretera Azua-Barahona hasta Loma del Curro (MES, BS, BT, BH, BN) y la segunda en Puerto Alejandro, en hondonadas secas de la vertiente sur de SMG para la vegetación ribereña (VR), resultando en seis zonas de estudio correspondientes a seis de los tipos de vegetación 
descritos por García et al. (2007). Las zonas fueron muestreadas en cuatro expediciones, entre los meses de mayo a septiembre de 2014, coincidiendo con la temporada lluviosa. Se realizaron 24 recorridos tipo sendero en las dos áreas anteriormente referidas, de una hora de duración y longitud variable cada uno (cuatro por zona de estudio). Los muestreos se realizaron entre las 9:00 y las 17:00 horas. En cada recorrido se anotaron o colectaron las especies según la necesidad de determinación. Los ejemplares colectados fueron determinados en el Museo Nacional de Historia Natural Profesor Eugenio de Jesús Marcano, quedando depositados en su colección entomológica. La taxonomía sigue a Perez-Gelabert (2008) y a Sourakov y Zakharov (2011) para el género Calisto.

Análisis estadístico. Se estimó la riqueza $(\mathrm{S})$ como el número de especies presentes y la abundancia (A) como la cantidad de ejemplares. La diversidad de mariposas se abordó con los índices de equidad de Shannon ( $\left.H^{\prime}\right)$ y dominancia de Simpson ( $\lambda$ ) (Magurran, 2004). Mediante el coeficiente de Jaccard (J) se determinó la similitud entre las zonas de estudio (Moreno, 2001).

Para determinar la eficiencia de los muestreos y la aproximación al número real de especies de la comunidad, se realizaron curvas de acumulación de especies utilizando los estimadores no paramétricos Chao 1 y Chao 2, Bootstrap y Jackknife 2. El análisis de las curvas sigue los procedimientos de Jiménez-Valverde y Hortal (2003) y fueron desarrollados con los programas EstimateS 9.1.0 (Colwell, 2013) y Statistica versión 12. A los fines de la realización de los análisis estadísticos solo se tomaron en consideración las especies que se registraron en los transectos.

\section{RESULTADOS Y DISCUSIÓN}

Riqueza y abundancia. Mediante los transectos se registraron 2,916 individuos distribuidos en cinco familias, 14 subfamilias, 46 géneros y 64 especies, representando esta última cifra el 33.68\% de la mariposas diurnas registradas para La Hispaniola. Del total de especies, 12 son endémicas de la isla y 22 constituyen nuevos registros para esta localidad. De las especies documentadas, siete fueron avistadas fuera de los transectos, sumando 71 especies las registradas en este estudio (Tabla 1). Schwartz (1987) lista 76 y Bastardo (2012) agrega Greta diaphanus quisqueya (Fox, 1963) para la zona, con lo cual suman 77 el número de especies en la literatura. Con los 22 nuevos registros resultantes de este estudio, aumenta a 99 el número de especies documentadas para la Sierra Martín García, lo que representa 52.10\% de las conocidas para La Hispaniola. Esto acerca aún más el número de especies conocidas de la zona a las citadas para la Sierra de Neiba (114), sistema montañoso más próximo, al que se le atribuye el mismo origen geológico, denotando gran similitud en la riqueza de la fauna de lepidópteros de ambos lugares, coincidiendo con lo propuesto por Schwartz (1987).

La familia Nymphalidae mostró la mayor riqueza de especies $(26,42.18 \%)$, la mayoría (ocho) pertenecientes a la subfamilia Nymphalinae. La más abundante fue la familia Pieridae (Fig. 1) con 2,257 individuos (77.4\%). Este elevado valor coincide con el período de migración de piéridos en los meses de verano, incluyendo 607 individuos de Ascia monuste eubotea (Godart, 1819) avistados, en su mayoría, en VR. Este último resultado fue superior a lo encontrado por Schwartz (1987) en cuanto a la abundancia por especie se refiere. Estas fluctuaciones poblacionales son comunes para los piéridos. En esta familia encontramos la subfamilia Coliadinae con el mayor número de especies (17) y de individuos de todo el estudio. Dos registros nuevos para la SMG son notorios, Atlantea cryptadia Sommer \& Schwartz, 1980 y Battus zetides (Munroe, 1971), ambas consideradas como vulnerables por la Lista Roja Nacional (Ministerio de Medio Ambiente y Recursos Naturales de la República Dominicana, 2011). La primera es una especie rara de la cual solo se colectó una hembra en el bosque nublado; la segunda tiene una distribución más amplia en las montañas de la isla. 


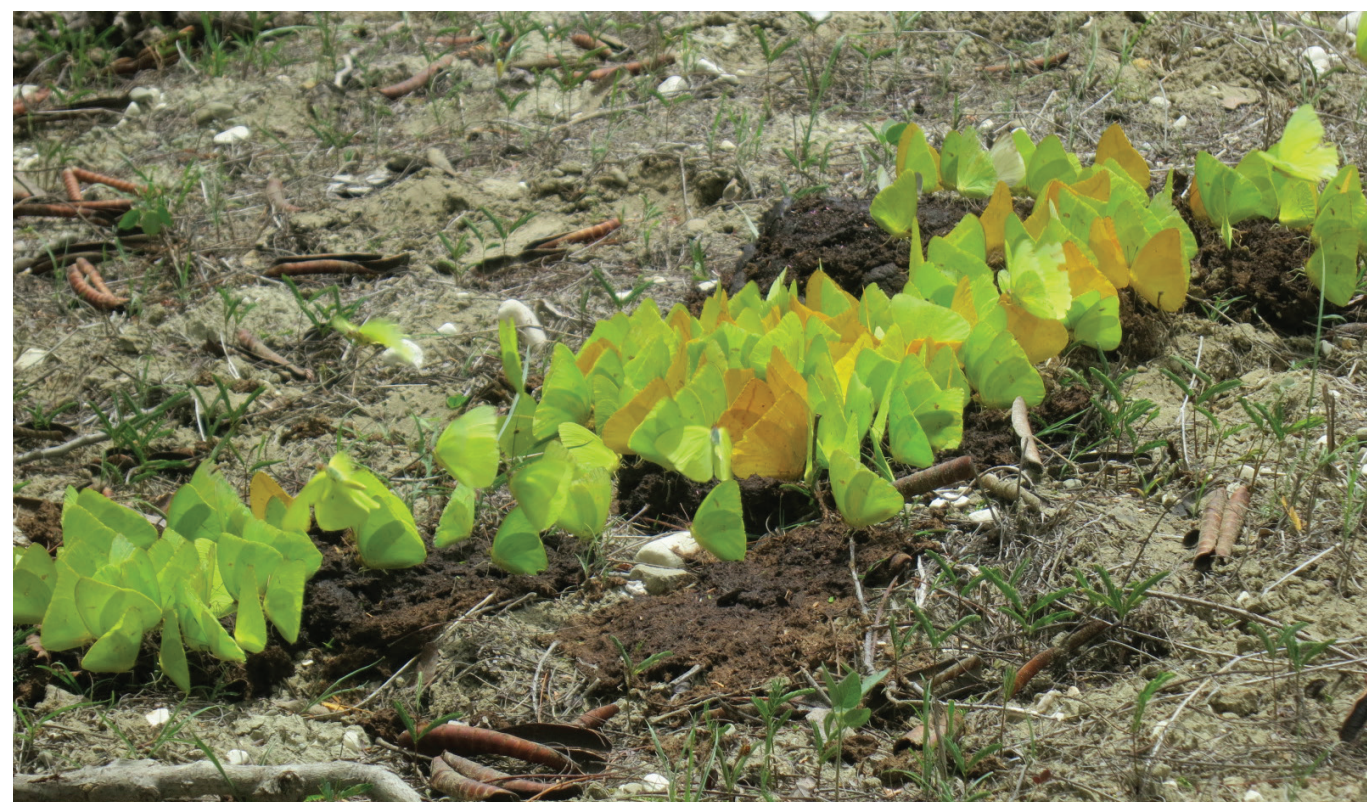

Figura 1. Phoebis agarithe y Phoebis Sennae, Monte Seco Espinoso Subtropical, Sierra Martín García.

Las zonas de mayor riqueza fueron BT y BH con 29 y 27 especies, respectivamente. El BH presentó la menor abundancia con apenas 148 individuos. Las especies con mayor número de individuos en BH fueron Archimestra teleboas (Ménétriés, 1832), Agraulis vanillae insularis Maynard, 1889, con 21 individuos cada una y Heliconius charitonia churchi Comstock \& Brown, 1950, con 20. De las 29 especies que se encontraron en el BT, 51.72\% pertenecen a la familia Nymphalidae, seguida de las familias Pieridae y Hesperiidae. Una distribución similar se observó en el BN, pero estuvo dentro de los lugares de menor riqueza (23 especies), en coincidencia con lo encontrado por Navarro (2002) y Bastardo (2007) en la Cordillera Central.

La vegetación ribereña presentó la menor riqueza de todo el estudio (21); tal vez un efecto de la sequía por la que atravesaba la SMG al momento de los muestreos. Los hábitats correspondientes a zonas más secas (MES y BS) mostraron un comportamiento distinto, siendo Pieridae la familia con mayor riqueza seguida de Nymphalidae.

Los resultados sobre la distribución de las familias de lepidópteros de SMG concuerdan con Schwartz (1987) para los hábitats mesófilos como BH y BN, donde la familia con mayor riqueza fue Nymphalidae, no obstante, en los resultados de aquel estudio Hesperiidae ocupa el segundo lugar en riqueza de especies, antes que Pieridae. Sin embargo, en nuestro estudio esta última familia fue la segunda de mayor riqueza en $\mathrm{BH}$ y BN. Por otro lado, los valores de abundancia en estos dos tipos de vegetación fueron los menores. En otras áreas de bosque nublado (Bastardo, 2007) se han descrito resultados similares, las condiciones climáticas características de los bosques nublados (bajas temperaturas y humedad relativa elevada), crean un ambiente poco adecuado para la mayoría de los lepidópteros.

La mayor abundancia se obtuvo en la vegetación ribereña con 829 individuos. Las especies más abundantes fueron A. m. eubotea con 512 y Kricogonia lyside (Godart, 1819) con 115 individuos. El valor de abundancia obtenido para la vegetación ribereña contrasta con el de riqueza (21 especies), siendo el menor para todo el estudio (Tabla 2). Estos comportamientos de la abundancia y riqueza se explican más adelante con los índices de diversidad. 
Las especies registradas para la zona y no encontradas en este estudio fueron 28: Aguna asander haitensis (Mabille \& Boullet, 1912), Anteos clorinde (Godart, 1824), Archaeoprepona demophoon insulicola (Godart, 1823), Asterocampa idyja idyja (Geyer, 1828), Burca stillmani Bell \& Comstock, 1948, Copaeodes stillmani (Bell \& Comstock, 1948), Cymaenes tripunctus (Herrich-Schäffer, 1865), Danaus cleophile (Godart, 1819), Epargyreus spanna Evans, 1952, Eunica tatila tatilista Kaye, 1926, Gesta gesta (Herrich-Schäffer, 1863), Greta diaphanus quisqueya (Fox, 1963), Hesperia nabokovi (Bell \& Comstock, 1948), Hesperia nabokovi (Bell \& Comstock, 1948), Hylephila phyleus (Drury, 1773), Hypanartia paullus (Fabricius, 1793), Junonia genoveva (Cramer, 1780), Libytheana terena (Godart, 1819), Marpesia eleuchea dospassosi (Munroe, 1971), Polygonus leo leo (Gmelin, 1790), Pyrgus crisia HerrichSchäffer, 1865, Pyrisitia euterpiformis (Munroe, 1947), Pyrisitia leuce memulus (Butler, 1871), Pyrrhocalles antiqua antiqua (Herrich-Schäffer, 1863), Siproeta stelenes stelenes (Linnaeus, 1758), Strymon bazochii (Godart, 1824), Strymon columella (Fabricius, 1793), Strymon toussainti (Comstock \& Huntington, 1943), Urbanus proteus domingo (Scudder, 1872).

Tabla 1. Lista de especies registradas en Parque Nacional Sierra Martín García y su distribución por tipo de vegetación.

\begin{tabular}{|c|c|c|c|c|c|c|}
\hline TÁXONES & VR & MES & BS & BT & $\mathrm{BH}$ & $\mathrm{BN}$ \\
\hline \multicolumn{7}{|l|}{ HESPERIIDAE } \\
\hline \multicolumn{7}{|l|}{ HESPERIINAE } \\
\hline Choranthus melissa Gali, 1983 & & & & & $\mathrm{X}$ & \\
\hline Synapte malitiosa adoceta Schwartz \& Sommer, 1986 & & & $\mathrm{X}$ & & & \\
\hline Ochlodes batesi (Bell, 1935) & & & & & & $\mathrm{X}$ \\
\hline Wallengrenia otho druryi (Latreille, 1824) & & & & & & $\mathrm{X}$ \\
\hline Panoquina ocola Johnson \& Matusik, 1988 & & & & $\mathrm{X}$ & & \\
\hline \multicolumn{7}{|l|}{ PYRGINAE } \\
\hline Burca hispaniolae Bell \& Comstock, 1948 & $\mathrm{X}$ & & & $\mathrm{X}$ & $\mathrm{X}$ & \\
\hline Ephyriades zephodes (Hübner, 1825) & $\mathrm{X}$ & & & $\mathrm{X}$ & $\mathrm{X}$ & \\
\hline Pyrgus oileus (Linnaeus, 1767)* & & & $\mathrm{X}$ & & $X$ & $\mathrm{X}$ \\
\hline Urbanus dorantes cramptoni Comstock, 1944 & & & & $\mathrm{X}$ & & $\mathrm{X}$ \\
\hline \multicolumn{7}{|l|}{ NYMPHALIDAE } \\
\hline \multicolumn{7}{|l|}{ BIBLIDINAE } \\
\hline Archimestra teleboas (Ménétriés, 1832) & & & & $\mathrm{X}$ & $\mathrm{X}$ & $\mathrm{X}$ \\
\hline Eunica monima (Cramer, 1782) & $\mathrm{X}$ & & & & & \\
\hline Dynamine serina zetes (Ménétriés, 1832)* & & & & $\mathrm{X}$ & & \\
\hline Hamadryas amphichloe diasia (Fruhstorfer, 1916) & $\mathrm{X}$ & $\mathrm{X}$ & $\mathrm{X}$ & $\mathrm{X}$ & $X$ & \\
\hline Myscelia aracynthia Dalman, 1823 & & & & & $\mathrm{X}$ & \\
\hline \multicolumn{7}{|l|}{ CHARAXINAE } \\
\hline Memphis verticordia Hübner, 1831 & $\mathrm{X}$ & & & $\mathrm{X}$ & $\mathrm{X}$ & \\
\hline \multicolumn{7}{|l|}{ DANAINAE } \\
\hline Anetia briarea briarea (Godart, 1819) & & & & & $\mathrm{X}$ & $\mathrm{X}$ \\
\hline Anetia pantheratus pantheratus (Martyn, 1797) & & & & & & $\mathrm{X}$ \\
\hline
\end{tabular}


Tabla 1 (continuación).

\begin{tabular}{|c|c|c|c|c|c|c|}
\hline TÁXONES & VR & MES & $\mathrm{BS}$ & $\mathrm{BT}$ & $\mathrm{BH}$ & $\mathrm{BN}$ \\
\hline Danaus cleophile (Godart, 1819) & & & $\mathrm{X}$ & & $\mathrm{X}$ & $\mathrm{X}$ \\
\hline Danaus eresimus tethys Forbes, 1944* & & $\mathrm{X}$ & $\mathrm{X}$ & & & \\
\hline Danaus gilippus cleothera (Godart, 1819) & & & & $\mathrm{X}$ & & \\
\hline Danaus plexippus megalippe (Hübner, 1826) & & & & & & $\mathrm{X}$ \\
\hline Lycorea halia cleobaea (Godart, 1819) & & & & & $\mathrm{X}$ & \\
\hline \multicolumn{7}{|l|}{ HELICONIINAE } \\
\hline Agraulis vanillae insularis Maynard, $1889^{*}$ & $\mathrm{X}$ & & $\mathrm{X}$ & $\mathrm{X}$ & $\mathrm{X}$ & $\mathrm{X}$ \\
\hline Dryas iulia fucatus (Boddaert, 1783) & $\mathrm{X}$ & $\mathrm{X}$ & $\mathrm{X}$ & $\mathrm{X}$ & $\mathrm{X}$ & \\
\hline Heliconius charitonia churchi Comstock \& Brown, 1950 & $\mathrm{X}$ & & $\mathrm{X}$ & $\mathrm{X}$ & $\mathrm{X}$ & $\mathrm{X}$ \\
\hline \multicolumn{7}{|l|}{ LIMENITIDINAE } \\
\hline Adelpha gelania gelania (Godart, 1824) & & $\mathrm{X}$ & & $\mathrm{X}$ & & \\
\hline \multicolumn{7}{|l|}{ NYMPHALINAE } \\
\hline Atlantea cryptadia Sommer \& Schwartz, 1980* & & & & & & $\mathrm{X}$ \\
\hline Anthanassa frisia (Poey, 1832)* & & & & & $\mathrm{X}$ & $\mathrm{X}$ \\
\hline Anartia jatrophae saturata (Staudinger, 1884)* & $\mathrm{X}$ & & $\mathrm{X}$ & & $\mathrm{X}$ & \\
\hline Anartia lytrea (Godart, 1819)* & $\mathrm{X}$ & & & & & \\
\hline Antillea pelops pelops (Drury, 1773) & & & & & $\mathrm{X}$ & \\
\hline Anaea troglodyta (Fabricius, 1775) & $\mathrm{X}$ & & $\mathrm{X}$ & & $\mathrm{X}$ & \\
\hline Euptoieta hegesia (Cramer, 1779) & & $\mathrm{X}$ & $\mathrm{X}$ & & & \\
\hline Historis odius odius (Fabricius, 1775) & & & & & $\mathrm{X}$ & \\
\hline Junonia evarete (Cramer, 1779)* & & $\mathrm{X}$ & & & & \\
\hline Vanessa virginiensis (Drury, 1773) & & & & & & $\mathrm{X}$ \\
\hline \multicolumn{7}{|l|}{ SATYRINAE } \\
\hline Calisto confusa Lathy, 1899 & & & & & $\mathrm{X}$ & $\mathrm{X}$ \\
\hline Calisto franciscoi Gali, 1985 & & $\mathrm{X}$ & & & & \\
\hline Calisto obscura Michener, 1943 & & & & $\mathrm{X}$ & $\mathrm{X}$ & $\mathrm{X}$ \\
\hline \multicolumn{7}{|l|}{ LYCAENIDAE } \\
\hline \multicolumn{7}{|l|}{ POLYOMMATINAE } \\
\hline Brephidium exilis (Herrich-Schäffer, 1862) & $\mathrm{X}$ & & & & & \\
\hline Cyclargus ammon noeli (Comstock \& Huntington, 1943) & $\mathrm{X}$ & $\mathrm{X}$ & $\mathrm{X}$ & $\mathrm{X}$ & $\mathrm{X}$ & \\
\hline Hemiargus hanno ceraunus (Fabricius, 1793) & $\mathrm{X}$ & $\mathrm{X}$ & & & & $\mathrm{X}$ \\
\hline Leptotes cassius theonus (Lucas, 1857) & & & $\mathrm{X}$ & & & $\mathrm{X}$ \\
\hline $\begin{array}{l}\text { Pseudochrysops bornoi bornoi (Comstock \& Huntington, } \\
\text { 1943)* }\end{array}$ & & $\mathrm{X}$ & & & & \\
\hline \multicolumn{7}{|l|}{ TECHLINAE } \\
\hline Strymon acis (Drury, 1773) & & & & & & $\mathrm{X}$ \\
\hline \multicolumn{7}{|l|}{ PAPILIONIDAE } \\
\hline PAPILIONINAE & & & & & & \\
\hline
\end{tabular}


Tabla 1 (continuación).

\begin{tabular}{|c|c|c|c|c|c|c|}
\hline TÁXONES & VR & MES & $\mathrm{BS}$ & BT & $\mathrm{BH}$ & $\mathrm{BN}$ \\
\hline Battus polydamas polycrates (Hopffer, 1865) & & & & $\mathrm{X}$ & & \\
\hline Battus zetides (Munroe, 1971)* & & & & & $\mathrm{X}$ & \\
\hline Heraclides androgeus epidaurus (Godman \& Salvin, 1890) & & & & & & $\mathrm{X}$ \\
\hline Heraclides aristodemus aristodemus Esper, 1794 & & & & $\mathrm{X}$ & $\mathrm{X}$ & \\
\hline Heraclides machaonides (Esper, 1796) & & & & $\mathrm{X}$ & & \\
\hline \multicolumn{7}{|l|}{ PIERIDAE } \\
\hline \multicolumn{7}{|l|}{ COLIADINAE } \\
\hline Aphrissa statira hispaniolae (Munroe, 1947)* & & $\mathrm{X}$ & & $\mathrm{X}$ & & \\
\hline Aphrissa orbis browni (Munroe, 1947)* & & $\mathrm{X}$ & $\mathrm{X}$ & & & \\
\hline Aphrissa godartiana godartiana (Swainson, 1821) & $\mathrm{X}$ & & & $\mathrm{X}$ & & $\mathrm{X}$ \\
\hline Abaeis nicippe (Cramer, 1782)* & & $\mathrm{X}$ & & & & \\
\hline Anteos maerula (Fabricius, 1775) & & & $\mathrm{X}$ & $\mathrm{X}$ & & \\
\hline Eurema daira palmira (Poey, 1852)* & $\mathrm{X}$ & $\mathrm{X}$ & & & & \\
\hline Eurema elathea elathea (Cramer, 1777)* & & $\mathrm{X}$ & $\mathrm{X}$ & & & \\
\hline Eurema nise larae (Herrich-Schäffer, 1862)* & & & & & $\mathrm{X}$ & $\mathrm{X}$ \\
\hline Eurema priddyi priddyi (Lathy, 1898)* & & $\mathrm{X}$ & & & & $\mathrm{X}$ \\
\hline Pyrisitia pyro (Godart, 1819) & & $\mathrm{X}$ & & $\mathrm{X}$ & $\mathrm{X}$ & \\
\hline Kricogonia lyside (Godart, 1819) & $\mathrm{X}$ & $\mathrm{X}$ & $\mathrm{X}$ & $\mathrm{X}$ & $\mathrm{X}$ & \\
\hline Phoebis agarithe antillia Brown, 1929* & $\mathrm{X}$ & $\mathrm{X}$ & $\mathrm{X}$ & $\mathrm{X}$ & & \\
\hline Phoebis editha (Butler, 1870)* & $\mathrm{X}$ & $\mathrm{X}$ & $\mathrm{X}$ & $\mathrm{X}$ & & $\mathrm{X}$ \\
\hline Phoebis philea thalestris (Illiger, 1801) & & & & & & $\mathrm{X}$ \\
\hline Phoebis sennae sennae (Linnaeus, 1758) & $\mathrm{X}$ & $\mathrm{X}$ & $\mathrm{X}$ & $\mathrm{X}$ & $\mathrm{X}$ & \\
\hline Pyrisitia lisa euterpe (Ménétriés, 1832) & $\mathrm{X}$ & $\mathrm{X}$ & $\mathrm{X}$ & & & $\mathrm{X}$ \\
\hline Zerene cesonia cynops (Butler, 1873)* & & & & $\mathrm{X}$ & & \\
\hline \multicolumn{7}{|l|}{ PIERINAE } \\
\hline Ascia monuste eubotea (Godart, 1819) & $\mathrm{X}$ & $\mathrm{X}$ & $\mathrm{X}$ & $\mathrm{X}$ & $\mathrm{X}$ & \\
\hline Glutophrissa drusilla boydi (Comstock, 1943) & & $\mathrm{X}$ & $\mathrm{X}$ & $\mathrm{X}$ & $X$ & $\mathrm{X}$ \\
\hline Glutophrissa punctifera (d'Almeida, 1939)* & & & $\mathrm{X}$ & & & \\
\hline Ganyra josephina josephina (Godart, 1819) & & $\mathrm{X}$ & & & & \\
\hline
\end{tabular}

Diversidad. El BH mostró el valor más alto para el índice de equidad de Shannon: H' = 2.72 (Tabla 2), esto probablemente se deba a una mayor disponibilidad de recursos y un mejor estado de conservación de la zona con presencia de plantas hospederas de familias como Euphorbiaceae y Brassicaceae. Los menores valores fueron observados para VR (1.56), BS y MES (2.15). Por otro lado, el mayor valor para el índice de dominancia de Simpson se obtuvo en VR (0.41) y el menor valor en $\mathrm{BH}(0.08)$. Los valores del índice de dominancia de Simpson para zonas bajas (VR, MES y BS) hacen notar que pocas especies contribuyen a la elevada abundancia registrada, esto probablemente se deba a las condiciones climatológicas desfavorables que perjudican las especies sensibles al estrés ecológico provocadas por la prolongada sequía que afectó el área, 
a la que se suman unas esporádicas lluvias coincidentes con los muestreos en dos de los recorridos realizados en una de ellas (BS) y las conocidas migraciones de piéridos en verano. También hay que tomar en consideración el fuerte impacto sobre la vegetación en el bosque seco producto del corte de madera para distintos fines (García et al., 2007).

Tabla 2. Riqueza, abundancia y diversidad de mariposas.

\begin{tabular}{ccccccc}
\hline & VR & MES & BS & BT & BH & BN \\
\hline S & 21 & 25 & 25 & 29 & 27 & 23 \\
A & 829 & 760 & 697 & 332 & 148 & 150 \\
H' & 1.56 & 2.15 & 2.15 & 2.45 & 2.72 & 2.6 \\
$(\lambda)$ & 0.41 & 0.16 & 0.16 & 0.13 & 0.08 & 0.1 \\
\hline
\end{tabular}

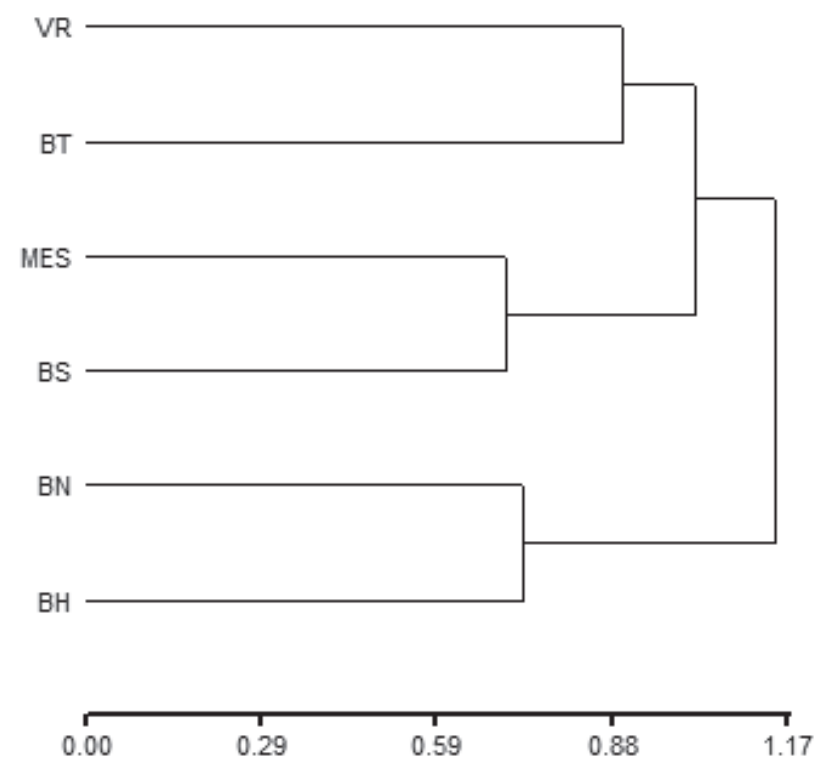

Figura 2. Dendrograma de los tipos de hábitats muestreados en función de la distribución de especies.

Similitud. El índice de similitud de Jaccard mostró la existencia de tres grupos o comunidades de mariposas en función de la zonificación altitudinal de la vegetación (Fig. 2). Los bosques de zonas bajas, BS y MES, fueron los más semejantes entre sí (30\%) en su composición de especies de lepidópteros (Tabla 3). A medida que se asciende en altitud esta relación entre comunidades se hace menor, resultando en un segundo grupo formado por los bosques húmedo y nublado, con una similitud de 28\%. El tercer grupo define los tipos de vegetación en donde existen influencias de las comunidades vegetales vecinas como la vegetación ribereña y los bosques de transición, aunque con una relación un tanto baja (10\%). Los hábitats con menor afinidad fueron MES y $\mathrm{BH}$, con apenas $3 \%$ de similitud. La diferente disponibilidad de recursos para adultos y larvas, además de factores climáticos como la temperatura, precipitación y velocidad del viento, podrían estar influyendo en estas diferencias, aunque estos parámetros no se midieron. 


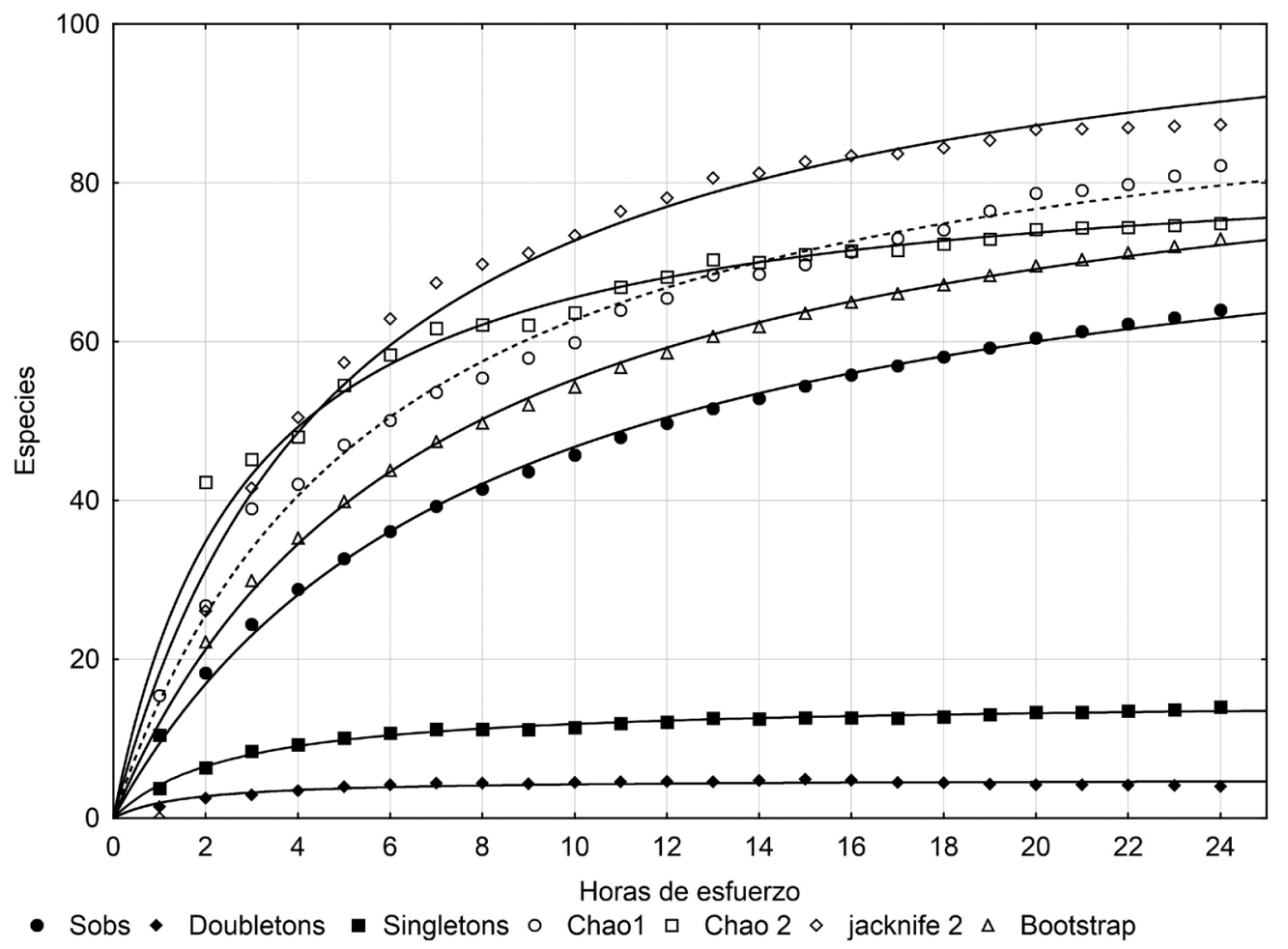

Figura 3. Curva de acumulación de especies.

Tabla 3. Matriz de similitud de Jaccard.

\begin{tabular}{ccccccc}
\hline & BH & BN & VR & BS & BT & MES \\
\hline BH & 1.00 & & & & & \\
BN & 0.28 & 1.00 & & & & \\
VR & 0.11 & 0.00 & 1.00 & & & \\
BS & 0.24 & 0.13 & 0.12 & 1.00 & & \\
BT & 0.15 & 0.09 & 0.10 & 0.06 & 1.00 & \\
MES & 0.03 & 0.08 & 0.10 & 0.30 & 0.08 & 1.00 \\
\hline
\end{tabular}

Curva de acumulación de especies. El modelo que se utilizó para analizar los datos (Simplex and Quasi Newton) tuvo un ajuste del 99.76\%. La curva no alcanzó la asíntota, es decir, el esfuerzo de muestreo fue insuficiente para registrar la totalidad de las especies esperadas (Fig. 3). La proporción de la fauna registrada fue 76.37\% (64 especies), representando $77.86 \%$ y $73.26 \%$ de las especies estimadas por Jacknife2 y Chao 1 respectivamente. La proporción de especies observadas podría considerarse bastante alta de acuerdo a literatura referenciada (Jiménez-Valverde y Hortal, 2003). Chao 2 y Bootstrap estimaron cifras más conservadoras 75 y 73 especies. Se espera que se sigan agregando especies, haciéndose evidente en la proporción de especies documentadas de la familia Hesperiidae, que solo alcanzó el 35\% de especies registradas por Schwartz (1987). Para documentar el 95\% de las especies sería necesario realizar 
un esfuerzo de muestreo de 150.39 horas. Se hace necesario continuar las exploraciones por otras rutas de acceso y temporadas diferentes del año para obtener un conocimiento más acabado de la fauna de lepidópteros del Parque Nacional Sierra Martín García

\section{AGRADECIMIENTOS}

Al Museo Nacional de Historia Natural "Prof. Eugenio de Jesús Marcano” por el auspicio y financiamiento de este estudio. A Brígido Peguero, Jardín Botánico Nacional Rafael M. Moscoso, por sus recomendaciones sobre las rutas de las expediciones en relación a la vegetación presente. Ángel Pimentel, Encargado de la Unidad de Biometría del Instituto Dominicano de Investigaciones Agropecuarias y Forestales (IDIAF) por su asesoría en lo concerniente a los análisis estadísticos. Carlos Suriel, por sus recomendaciones para la realización del estudio. Hodali Almonte, Gabriel de los Santos y Solanlly Carrero por sus sugerencias al manuscrito y Cristian Marte por su ayuda en los viajes de campo.

\section{LITERATURA CITADA}

Abreu, D. y K. Guerrero. 1997. Evaluación ecológica integral Parque Nacional del Este, República Dominicana: The Nature Conservancy, United States Agency for International Development, Banco Interamericano de Desarrollo, Dirección Nacional de Parques y Fondo Integrado por la Naturaleza.133 pp.

Bastardo, R. 2007. Diversidad de Lepidoptera: Rhopalocera (mariposas diurnas) del Parque Nacional Armando Bermúdez por medio de una Evaluación Ecológica Integrada, 61-92 pp. En: F. Núñez, ed. Evaluación Ecológica Integrada del Parque Nacional Parque Nacional Armando Bermúdez. Secretaría de Estado de Medio Ambiente y Recursos Naturales/ Fundación Moscoso Puello. 164 pp.

Bastardo, R. 2012. Nuevas localidades para Greta diaphanus quisqueya (Fox, 1963) у Papilio demoleus Linneus, 1758 (Lepidoptera: Papilionoidea) en La Hispaniola. Novitates Caribaea, 5:103-109.

Colwell, R. K. 2013. EstimateS: Statistical estimation of species richness and shared species from samples. Version 9.1.0. Persistent URL < purl.oclc.org/estimates>.

De La Fuente, S. 1976. Geografía Dominicana. Editorial Colegial Quisqueyana, Santo Domingo, República Dominicana, 165 pp.

García, R., B. Peguero, T. Clase, A. Veloz, F. Jiménez y M. Mejía. 2007. Flora y Vegetación de las zonas áridas de la Sierra Martín García, República Dominicana. Moscosoa, 15: 5-60.

Jiménez-Valverde, A. y J. Hortal. 2003. Las curvas de acumulación de especies y la necesidad de evaluar la calidad de los inventarios biológicos. Revista Ibérica de Aracnología, 8: 151-161.

Magurran, A. E. 2004. Measuring biological diversity. Oxford: Blackwell Publishing. 215 pp.

Ministerio de Medio Ambiente y Recursos Naturales de la República Dominicana, 2011. Lista de Especies en Peligro de Extinción, Amenazadas y Protegidas de la República Dominicana (Lista Roja). 44 pp. 
Moreno, C. E. 2001. Métodos para medir la biodiversidad. M\&T-Manuales y Tesis SEA, vol.1. Zaragoza, 84 pp.

Myers, N., R. A. Mittemeier, C. G. Mittermeier, G. A. B. da Fonseca y J. Kent. 2000. Biodiversity hotspots for conservation priorities. Nature, 403: 853-858.

Navarro, S. 2002. Mariposas del Parque Nacional Juan Bautista Pérez Rancier (Valle Nuevo), 99-107. En: F. Núñez, ed. Evaluación Ecológica Integrada Parque Nacional Juan B. Pérez Rancier (Valle Nuevo). Secretaría de Estado de Medio Ambiente y Recursos Naturales/ Fundación Moscoso Puello. 147 pp.

Núñez Águila, R. y A. Barro Cañamero. 2012. A list of Cuban Lepidoptera (Arthropoda: Insecta). Zootaxa, 3384: 1-59.

Perdomo, L., Y. Arias, Y. Léon y D. Wege. 2010. Áreas Importantes para la Conservación de las Aves en la República, Dominicana. Grupo Jaragua y el Programa IBA-Caribe de BirdLife International: Santo Domingo, República Dominicana. 84 pp.

Perez-Gelabert, D. E. 2008. Arthropods of Hispaniola (Dominican Republic and Haiti): a checklist and bibliography. Zootaxa, 1831: 1-530.

Powell, R. y S. J. Incháustegui. 2009. Conservation of the herpetofauna of the Dominican Republic. Applied Herpetology, 6: 103-122.

Schwartz, A. 1983. Haitian butterflies, University of Florida Press, Gainesville, 69 pp.

Schwartz, A. 1987. Butterflies of the Sierra Martín García, República Dominicana. Caribbean Journal of Science, 23: 418-431.

Schwartz, A. 1989. The butterflies of Hispaniola, University of Florida Press, Gainesville, $580 \mathrm{pp}$.

Smith D. S., L. D. Miller, J. Y. Miller. 1994. The Butterflies of the West Indies and South Florida. Oxford University Press, Oxford, 264 pp.

Sourakov, A. y E. V. Zakharov. 2011. "Darwin's butterflies"? DNA barcoding and the radiation of the endemic Caribbean butterfly genus Calisto (Lepidoptera, Nymphalidae, Satyrinae). Comparative Cytogenetics, 5: 191-210.

Takizawa, H., S. Medrano y D. Veloz. 2003. Guía de mariposas diurnas de La Hispaniola. Museo Nacional de Historia Natural y Agencia de Cooperación Internacional del Japón. 111 pp.

Troncoso, B. M. 1986. Regiones Geomorfológicas de La Española o de Santo Domingo. Editora Universitaria-UASD. Santo Domingo, República Dominicana. 81pp.

[Recibido: 10 de julio, 2014. Aceptado para publicación: 20 de septiembre, 2014] 